\title{
Endogenous reference points in bargaining
}

\author{
Emin Karagözoğlu1,2 - Kerim Keskin ${ }^{3}$
}

Received: 18 July 2017 / Accepted: 22 April 2018 / Published online: 27 April 2018

(C) Springer-Verlag GmbH Germany, part of Springer Nature 2018

\begin{abstract}
We allow the reference point in (cooperative) bargaining problems with a reference point to be endogenously determined. Two loss averse agents simultaneously and strategically choose their reference points, taking into consideration that with a certain probability they will not be able to reach an agreement and will receive their disagreement point outcomes, whereas with the remaining probability an arbitrator will distribute the resource by using (an extended) Gupta-Livne bargaining solution (Gupta and Livne in Manag Sci 34:1303-1314, 1988). The model delivers intuitive equilibrium comparative statics on the breakdown probability, the loss aversion coefficients, and the disagreement point outcomes.
\end{abstract}

Keywords Bargaining problem · Gupta-Livne solution · Loss aversion · Prospect theory $\cdot$ Reference points

\section{Introduction}

The reference point idea stipulates that individuals evaluate outcomes with respect to a reference outcome in many decision making situations. The source of a reference point may be culture, historical precedents, norms, laws, regulations, environmental

Kerim Keskin

kerim.keskin@khas.edu.tr

Emin Karagözoğlu

karagozoglu@bilkent.edu.tr

1 Department of Economics, Bilkent University, 06800 Çankaya, Ankara, Turkey

2 CESifo, Poschingerstr. 5, 81679 Munich, Germany

3 Department of Economics, Kadir Has University, 34083 Fatih, İstanbul, Turkey 
cues, aesthetic reasons, focal points, or the values of relevant economic parameters. In negotiations, Ashenfelter and Bloom (1984) pointed out that the previous agreement constitutes a natural reference point, even when it expired. Similarly, Bazerman (1985) reported in a survey experiment that arbitrators' judgments of a fair labor contract are heavily influenced by previous contractual conditions.

Following an increased interest in the experimental investigation of reference point effects in negotiations, there has been a number of studies which report that reference points - in the form of existing contracts, reservation prices, expired contracts, historical contractual conditions, informal agreements, fairness norms-significantly influence the whole bargaining process and the negotiated agreement (see Blount et al. 1996; Kristensen and Gärling 2000; Gächter and Riedl 2005; Bartling and Schmidt 2015; Karagözoğlu and Riedl 2015; Bolton and Karagözoğlu 2016 among others).

Motivated by the criticisms challenging the scientific value of theories that use exogenously given reference points to explain individual behavior (e.g., with an appropriate choice of a reference point, any observed behavior can be explained), there also has been a surge in the number of studies that allow the reference point to be endogenously determined (see Shalev 2000, 2002; Compte and Jehiel 2004; Köszegi and Rabin 2006; Vartiainen 2007; Hyndman 2011; and Driesen et al. 2012 among others). However, despite the fact that the reference point idea is not new in the cooperative bargaining literature (see Brito et al. 1977; Thomson 1981), endogenous emergence of reference points is still an understudied topic in this literature. This paper aims to contribute to a line of research modeling the emergence of reference points in cooperative bargaining problems.

The reference point is endogenously determined in our model. This work shares a similar spirit with two strands of literature. The first one is commitment in strategic bargaining (see Muthoo 1992; Ellingsen and Miettinen 2008, 2014 among others). In Muthoo (1992), before the bargaining stage, each agent simultaneously and strategically makes a commitment by announcing that offers below a certain level will not be accepted. If an agent receives an outcome lower than his commitment level, then he experiences disutility (i.e., revoking cost). Accordingly, commitment behavior directly influences the bargaining outcome. The other strand of literature focuses on cooperative bargaining models with loss averse agents (see Shalev 2002; Driesen et al. 2011 among others). In Shalev (2002), if an agent receives an outcome below his reference point outcome, then he perceives this as a loss and experiences disutility. Since the author utilized the Nash bargaining solution in his study, the reference point only indirectly affects the bargaining outcome through its influence on the bargaining set.

For the sake of expositional simplicity, we stick to one of these two interpretations. In particular, we formulate our model along similar lines with Shalev (2002) and subscribe to the "reference point-loss aversion" interpretation. Two major differences from Shalev (2002) are worth mentioning here. First, we introduce a pre-bargaining game in which agents simultaneously and strategically choose their reference point outcomes taking into consideration that with an exogenously given probability they will not be able to reach an agreement and will receive their disagreement point outcomes, whereas with the remaining probability an arbitrator will distribute the resource among them. Second, we assume that the arbitrator employs (an extended 
version of) the bargaining solution proposed by Gupta and Livne (1988). Accordingly, the reference point has a direct influence on the bargaining outcome. We are closer to Muthoo (1992)'s model in these two dimensions: agents make commitments, which in turn become their reference points, and the arbitrator considers these commitment levels by employing a bargaining solution that would not give agents less than what they committed to. As in Shalev (2002), both agents are loss averse. This induces a trade-off for the agents while they are choosing their reference point outcomes: a high reference point is not desirable in anticipation of a disagreement (due to reference-dependent utility), whereas it is desirable in anticipation of an agreement (a higher reference point, ceteris paribus, leads to a higher share). Consequently, the equilibrium reference point outcomes depend on the breakdown probability, the loss aversion coefficients, and the disagreement point outcomes.

In our main result, we show that multiple equilibrium reference points are possible. For sufficiently high values of the exogenously given breakdown probability and the loss aversion coefficients, the equilibrium reference point outcome coincides with the disagreement point outcome. When these parameters are lower than certain critical values, the equilibrium on the disagreement point vanishes, but there appears another corner solution in which one agent chooses his disagreement point outcome as his reference point outcome, whereas the other agent claims the remaining resource. In addition to these, there may exist a continuum of equilibrium reference point outcomes on the frontier. The size of the set of equilibrium reference point outcomes on the frontier depends on multiple model parameters in an intuitive fashion, which will be discussed in greater detail in Sect. 3. It is the presence of the trade-off generated by loss aversion and the possibility of breakdown, coupled with the convexity of the Gupta-Livne solution (in the reference point outcome), which leads to these extremal equilibria.

We are aware of two papers, which are arguably similar to our study. Vartiainen (2007) studied bargaining problems without a disagreement point and characterized an extended solution concept which determines a disagreement outcome along with the solution. Bozbay et al. (2012) assumed that the endogenously determined disagreement outcome plays the role of agents' mental reference points, representing their common beliefs of what would happen if they fail to reach an agreement. It is worth noting that, in both of these papers, reference points emerge during the bargaining stage, are only well-defined for the proposed bargaining solutions, hence are essentially different from the reference points described by Gupta and Livne (1988).

Our model shares the flavor of Luce and Raiffa (1957)'s idea of a reference point emerging as an outcome of a strategic interaction. To the best of our knowledge, ours is the first study in which the reference point in a cooperative bargaining model is endogenously and optimally determined in a strategic pre-bargaining game. The current work can also be thought of as a belated follow-up on Gupta and Livne (1988), since the source of the reference point was unexplained there and explaining its emergence was left for future research.

This paper is organized as follows. In Sect. 2, we formulate the model; and in Sect. 3, we present a detailed analysis of Nash equilibrium. Section 4 concludes. 


\section{The model}

\subsection{The Gupta-Livne solution}

A bargaining set is a non-empty, closed, convex, and comprehensive set $\mathcal{S} \subset \mathbb{R}^{2}$ bounded from above, which consists of all utility vectors that can be achieved by the agents. The disagreement point $D \in \mathcal{S}$ represents the utilities received by the agents in case no agreement is reached. The ideal (or utopia) point is denoted by $U \equiv a(\mathcal{S}, D)$, where $U_{i} \equiv a_{i}(\mathcal{S}, D)=\max \left\{t \in \mathbb{R} \mid\left(t, D_{-i}\right) \in \mathcal{S}\right\}$; and it gives, for each agent, the maximum utility level that can be reached in an individually rational agreement. A bargaining problem is a pair $(\mathcal{S}, D)$ such that there exists some $x \in \mathcal{S}$ with $x \gg D .^{1}$ A bargaining problem with a reference point is a triple $(\mathcal{S}, D, R)$ where the reference point is such that $R \in \mathcal{S}$ and $R \geq D$.

Let $\Sigma^{2}$ be the family of all bilateral bargaining problems with a reference point. A solution concept on $\Sigma^{2}$ is a function $\phi$ that associates with each triple $(\mathcal{S}, D, R) \in \Sigma^{2}$ a unique outcome $\phi(\mathcal{S}, D, R) \in \mathcal{S}$.

Now, the Gupta-Livne solution $(G L)$ can be formally defined as follows.

Definition 1 For every $(\mathcal{S}, D, R) \in \Sigma^{2}$,

$$
G L(\mathcal{S}, D, R) \equiv \delta^{*} a(\mathcal{S}, D)+\left(1-\delta^{*}\right) R
$$

where $\delta^{*}=\max \{\delta \in[0,1] \mid(\delta a(\mathcal{S}, D)+(1-\delta) R) \in \mathcal{S}\}$.

$G L$ proposes the maximum point in the bargaining set and on the line segment connecting the ideal point, $a(\mathcal{S}, D)$, and the reference point, $R$. This is equivalent to saying that $G L$ chooses the maximum individually rational utility profile at which each agent's utility gain from his reference point has the same proportion to the utility difference between his ideal point and his reference point. As mentioned earlier, we employ an extended version of $G L$ in this paper. The difference between our extended version and the original solution is small: (i) we allow $R$ to be on the weak Pareto frontier and assume that if so, the extended $G L$ proposes $R$ as the agreement; and (ii) we allow $R$ to be outside the bargaining set and assume that if so, the extended $G L$ prescribes $D$ as the outcome. ${ }^{2}$

\subsection{Endogenizing the reference point}

Kahneman and Tversky (1979) formulated prospect theory as a descriptive model of individual behavior. Including loss aversion and reference dependence as its two key aspects, prospect theory makes a clear distinction between a tangible/physical resource and an intangible value or utility. Accordingly, each individual evaluates an earning below his reference point as a loss and an earning above his reference point

\footnotetext{
${ }^{1}$ For $x, y \in \mathbb{R}^{n}$, the vector inequalities are given as: $x \geq y, x>y$, and $x \gg y$.

2 Alternatively, one can capture the cases in (i) and (ii) via the strategic game form instead of by extending the bargaining solution. We chose the latter alternative for reader-friendliness.
} 
as a gain (i.e., reference dependence); and moreover, losses loom larger than gains (i.e., loss aversion). Both earnings and reference points are defined in terms of the tangible resource, whereas the satisfaction from possessing a portion of the resource is represented by an intangible prospect-theoretic utility. We now incorporate this type of reference-dependent preferences into the bargaining problem by introducing a tangible/physical resource to be divided between the agents such that the agents' utilities, which characterize the corresponding bargaining set, are derived with respect to their reference points.

There are two agents, and there is a perfectly divisible resource with size normalized to 1 . The disagreement point outcome for agent $i=1,2$ is denoted by $d_{i}$ such that $d_{1}+d_{2}<1$. There is an exogenously given breakdown probability $p \in(0,1)$ with which the resolution of conflict fails due to external factors. ${ }^{3}$ In such a case, the disagreement point outcome is realized. With the remaining probability, the bargaining outcome is determined using the extended $G L$. These are common knowledge. Here, we subscribe to a commonly used interpretation of cooperative bargaining solution concepts in the literature: there is an arbitrator who applies the solution concept in question to resolve the conflict between parties.

We are interested in the emergence of reference points in a bargaining problem. Hence, we now introduce the pre-bargaining game whose outcome influences the bargaining set and the corresponding salient points. In this pre-bargaining game, knowing that the extended $G L$ will be implemented with probability $1-p$, both agents simultaneously and strategically choose their reference point outcomes, $r_{1}$ and $r_{2} .{ }^{4}$ Agents' strategy sets are bounded from below by their disagreement point outcomes; i.e., $r_{i} \geq d_{i}$. In order to derive the corresponding bargaining set, we rely on the value function introduced by Shalev (2000). This function captures two key aspects of prospect theory preferences (see Kahneman and Tversky 1979): reference dependence and loss aversion. For agent $i=1,2$, the value function is defined as follows:

$$
V_{i}\left(x_{i}, r_{i}\right)= \begin{cases}x_{i} & \text { if } \quad x_{i} \geq r_{i} \\ x_{i}+\lambda_{i}\left(x_{i}-r_{i}\right) & \text { if } \quad x_{i}<r_{i}\end{cases}
$$

where $x_{i}$ is the amount of resource agent $i$ receives, $r_{i}$ is the reference point outcome of agent $i$, and $\lambda_{i}>0$ is the loss aversion coefficient of agent $i$. Note that the value function above implies a linear or piece-wise linear utility possibilities frontier (with one or two possible kinks) (see Fig. 1).

In this subsection, thus far, we used small letters to denote variables in terms of the resource. In the following, we utilize capital letters to represent the value/utility received. This way, the notation for the bargaining problem with an endogenous reference point will be consistent with the notation introduced in Sect. 2.1.

\footnotetext{
3 This is similar to Nash's probabilistic extension of his non-cooperative demand game where agents receive their disagreement point payoffs with probability $p$ and their claims with probability $1-p$ in case of a conflict (see Nash 1953).

4 Sarver (2012) also modeled agents who make a conscious and optimal choice of their reference points (in an individual decision making context).
} 


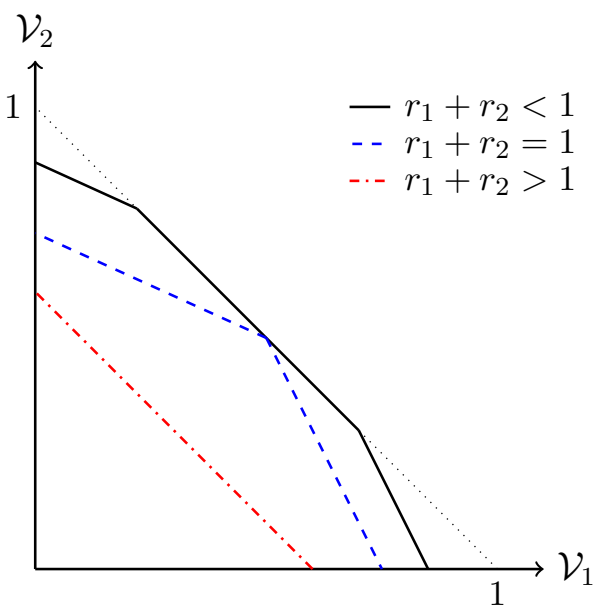

Fig. 1 Three possible types of bargaining sets

From this point onward, we use the term utility whenever we mean value as in the terminology of prospect theory. Given a reference point $r=\left(r_{1}, r_{2}\right) \geq\left(d_{1}, d_{2}\right)=d$, the disagreement point utility for agent $i=1,2$ is given by

$$
D_{i}(d, r) \equiv V_{i}\left(d_{i}, r_{i}\right)=d_{i}+\lambda_{i}\left(d_{i}-r_{i}\right)
$$

The ideal (or utopia) point utility for agent $i=1,2$ is given by

$$
U_{i}(d, r) \equiv V_{i}\left(1-d_{j}, r_{i}\right)= \begin{cases}1-d_{j} & \text { if } \quad r_{i} \leq 1-d_{j} \\ 1-d_{j}+\lambda_{i}\left(1-d_{j}-r_{i}\right) & \text { if } \quad r_{i}>1-d_{j}\end{cases}
$$

where $i \neq j$. For this, we assign agent $j$ his disagreement point outcome $d_{j}$ and calculate the utility of agent $i$ from having the remaining resource: $1-d_{j}$. Notice that the latter case " $r_{i}>1-d_{j}$ " is realized only when $r_{1}+r_{2}>1$, i.e., when agents choose mutually incompatible reference points. Moreover, the reference point utility for agent $i=1,2$ is

$$
R_{i}(d, r) \equiv V_{i}\left(r_{i}, r_{i}\right)=r_{i} .
$$

As mentioned above, the extended $G L$ is defined in such a way that the agents receive their reference point outcomes when $r_{1}+r_{2}=1$. Accordingly, if $r_{1}+r_{2} \leq 1$, the extended $G L$ gives a utility of $\mathcal{V}_{i}^{G L}$ to agent $i=1$, 2. Suppressing the dependence of the functions on $(d, r)$ when there is no risk of confusion, we find

$$
\mathcal{V}_{2}^{G L}=\frac{U_{2}-R_{2}}{U_{1}-R_{1}}\left(\mathcal{V}_{1}^{G L}-R_{1}\right)+R_{2}
$$

We know that for any Pareto optimal utility pair $\mathcal{V}(d, r)=\left(\mathcal{V}_{1}(d, r), \mathcal{V}_{2}(d, r)\right)$ satisfying $\mathcal{V}(d, r) \geq R$, we have $\mathcal{V}_{1}(d, r)+\mathcal{V}_{2}(d, r)=1$. Since $\left(\mathcal{V}_{1}^{G L}, \mathcal{V}_{2}^{G L}\right)$ is such a utility pair, this implies that 


$$
\mathcal{V}_{i}^{G L}=\frac{1-d_{j}-d_{i} r_{i}-r_{j}+d_{j} r_{j}}{2-d_{1}-d_{2}-r_{1}-r_{2}} \quad \text { where } i \neq j .
$$

What is left is to define $\mathcal{V}_{i}^{G L}$ for the remaining case, i.e., when $r_{1}+r_{2}>1$. For that, we recall that if the reference point is outside the bargaining set, then the extended $G L$ prescribes $D$ as the outcome. Accordingly,

$$
\mathcal{V}_{i}^{G L}=D_{i}=d_{i}+\lambda_{i}\left(d_{i}-r_{i}\right)
$$

for every $i=1,2$ in case $r_{1}+r_{2}>1 .^{5}$ In that regard, our pre-bargaining game resembles a divide-the-dollar game.

In our model, when choosing his reference point outcome, agent $i$ 's objective is to maximize

$$
p D_{i}(d, r)+(1-p) \mathcal{V}_{i}^{G L}(d, r) .
$$

We note once again that the presence of uncertainty poses a trade-off for the agents: a high reference point outcome is desirable in case of a resolution but not desirable in case of a breakdown.

\section{The results}

We now present a characterization of equilibrium reference point outcomes focusing on the set of pure strategy Nash equilibria.

Proposition 1 For the pre-bargaining game described above, the following statements are true:

(i) If

$$
p \geq \frac{1}{1+2 \lambda_{i}} \quad \text { for every } i=1,2
$$

(ii)(a) If

then $r^{*}=\left(d_{1}, d_{2}\right)$ is a Nash equilibrium.

$$
p \leq \frac{1}{1+2 \lambda_{2}}
$$

then $r^{*}=\left(d_{1}, 1-d_{1}\right)$ is a Nash equilibrium.

(ii)(b) If

$$
p \leq \frac{1}{1+2 \lambda_{1}}
$$

then $r^{*}=\left(1-d_{2}, d_{2}\right)$ is a Nash equilibrium.

(iii) If

$$
p<\frac{1}{1+\lambda_{i}} \text { for every } i=1,2
$$

\footnotetext{
${ }^{5}$ Nevertheless, $R$ will not be above the weak Pareto frontier in any equilibrium. We will formally argue this in the proof of Proposition 1.
} 
then $r^{*}=\left(r_{1}, 1-r_{1}\right)$ is a Nash equilibrium where

$$
d_{1}+\frac{p \lambda_{1}\left(1-d_{1}-d_{2}\right)}{1-p-p \lambda_{1}} \leq r_{1} \leq 1-d_{2}-\frac{p \lambda_{2}\left(1-d_{1}-d_{2}\right)}{1-p-p \lambda_{2}} .
$$

Proof We start with an important observation. Suppose that there exists an equilibrium reference point outcome $\left(r_{1}^{*}, r_{2}^{*}\right)$ such that $r_{1}^{*}+r_{2}^{*}>1$. Then, at least one of the agents can make himself better off by deviating to his disagreement point outcome; a contradiction. Hence, an equilibrium reference point outcome should satisfy $r_{1}^{*}+r_{2}^{*} \leq 1$.

Now, without loss of generality, we fix a strategy $\bar{r}_{2}$ for agent 2 and perform the best response analysis for agent 1 . The Eq. (3) can be rewritten as

$$
\mathcal{V}_{1}^{G L}=\frac{1-d_{2}-d_{1} r_{1}-\bar{r}_{2}+d_{2} \bar{r}_{2}}{2-d_{1}-d_{2}-r_{1}-\bar{r}_{2}}
$$

Then, $\mathcal{V}_{1}^{G L}$ is midpoint convex since

$$
\frac{\mathcal{V}_{1}^{G L}\left(d,\left(r_{1}, \bar{r}_{2}\right)\right)}{2}+\frac{\mathcal{V}_{1}^{G L}\left(d,\left(r_{1}^{\prime}, \bar{r}_{2}\right)\right)}{2} \geq \mathcal{V}_{1}^{G L}\left(d,\left(\frac{r_{1}+r_{1}^{\prime}}{2}, \bar{r}_{2}\right)\right)
$$

for every $r_{1}, r_{1}^{\prime}<1-\bar{r}_{2}$. Since $\mathcal{V}_{1}^{G L}$ is continuous in $r_{1}$ on the relevant domain, we conclude that $\mathcal{V}_{1}^{G L}$ is convex (see Delfour 2012).

Due to the convexity of $V_{1}^{G L}$, we know that agent 1 either chooses $d_{1}$ or $1-\bar{r}_{2}$ as his reference point outcome. That is, the optimization problem reduces to the comparison of the expected utility agent 1 receives when he chooses $d_{1}$, which is

$$
p d_{1}+(1-p) \mathcal{V}_{1}^{G L}\left(d,\left(d_{1}, \bar{r}_{2}\right)\right)
$$

and the expected utility agent 1 receives when he chooses $1-\bar{r}_{2}$, which is

$$
p d_{1}+p \lambda_{1}\left(d_{1}-1+\bar{r}_{2}\right)+(1-p) \mathcal{V}_{1}^{G L}\left(d,\left(1-\bar{r}_{2}, \bar{r}_{2}\right)\right)
$$

Considering (5), the Eqs. (6) and (7) above become

$$
p d_{1}+(1-p) \frac{1-d_{2}-d_{1}^{2}-\bar{r}_{2}+d_{2} \bar{r}_{2}}{2-2 d_{1}-d_{2}-\bar{r}_{2}}
$$

and

$$
p d_{1}+p \lambda_{1}\left(d_{1}-1+\bar{r}_{2}\right)+(1-p) \frac{1-d_{2}-d_{1}\left(1-\bar{r}_{2}\right)-\bar{r}_{2}+d_{2} \bar{r}_{2}}{1-d_{1}-d_{2}},
$$


respectively. Agent 1 prefers $d_{1}$ as his reference point outcome if the former is greater than or equal to the latter, that is, if

$$
\begin{aligned}
& \frac{\left(1-d_{1}-\bar{r}_{2}\right)\left[\left(d_{1}-d_{1} \bar{r}_{2}-1+d_{2}+\bar{r}_{2}-d_{2} \bar{r}_{2}\right)+d_{1}\left(1-d_{1}-d_{2}\right)\right]}{\left(1-d_{1}-d_{2}\right)\left(2-2 d_{1}-d_{2}-\bar{r}_{2}\right)} \\
& \geq-\frac{p \lambda_{1}\left(1-d_{1}-\bar{r}_{2}\right)}{1-p},
\end{aligned}
$$

which reduces to

$$
\frac{1-d_{1}-\bar{r}_{2}}{\left(1-d_{1}-\bar{r}_{2}\right)+\left(1-d_{1}-d_{2}\right)} \leq \frac{p \lambda_{1}}{1-p},
$$

noting that $1-d_{1}-\bar{r}_{2} \geq 0$ and $1-d_{1}-d_{2}>0$. Otherwise, agent 1 's best response would be to choose $1-\bar{r}_{2}$. Finally, by symmetry, similar arguments follow for agent 2 . As a result, the best response correspondence $B R_{i}$ for agent $i=1,2$ can be written as

$$
B R_{i}\left(r_{j}\right)= \begin{cases}d_{i} & \text { if } \quad \frac{1-d_{i}-r_{j}}{\left(1-d_{i}-r_{j}\right)+\left(1-d_{i}-d_{j}\right)} \leq \frac{p \lambda_{i}}{1-p} \\ 1-r_{j} & \text { if } \quad \frac{1-d_{i}-r_{j}}{\left(1-d_{i}-r_{j}\right)+\left(1-d_{i}-d_{j}\right)} \geq \frac{p \lambda_{i}}{1-p}\end{cases}
$$

It is worth noting that such a discontinuous best response correspondence is indeed an outcome of the convexity of $\mathcal{V}_{i}^{G L}$.

Accordingly, there exist three types of Nash equilibria. In particular, if for every agent $i=1,2: B R_{i}\left(d_{j}\right)=d_{i}$, then the strategy profile $r^{*}=\left(d_{1}, d_{2}\right)$ turns out to be a Nash equilibrium. This is satisfied, if for every $i=1,2$ :

$$
p \geq \frac{1}{1+2 \lambda_{i}}
$$

On the other hand, if for some $i=1,2$ :

$$
p \leq \frac{1}{1+2 \lambda_{i}}
$$

then there exists a corner solution in which agent $i$ chooses $1-d_{j}$ whereas agent $j$ chooses $d_{j}$.

Finally, there is an interval of Nash equilibria on the frontier satisfying $r_{1}^{*}+r_{2}^{*}=1$. To characterize this type of equilibria, we perform the following analysis. We say that the strategy profile $r^{*}=\left(r_{1}, 1-r_{1}\right)$ is an equilibrium if

$$
\begin{aligned}
\frac{r_{1}-d_{1}}{\left(r_{1}-d_{1}\right)+\left(1-d_{1}-d_{2}\right)} & \geq \frac{p \lambda_{1}}{1-p} \\
\frac{1-d_{2}-r_{1}}{\left(1-d_{2}-r_{1}\right)+\left(1-d_{1}-d_{2}\right)} & \geq \frac{p \lambda_{2}}{1-p}
\end{aligned}
$$

under the condition that $d_{1} \leq r_{1} \leq 1-d_{2}$, which follow from the fact that each agent's strategy set is bounded from below by his disagreement point outcome. These conditions reduce to 


$$
d_{1}+\frac{p \lambda_{1}\left(1-d_{1}-d_{2}\right)}{1-p-p \lambda_{1}} \leq r_{1} \leq 1-d_{2}-\frac{p \lambda_{2}\left(1-d_{1}-d_{2}\right)}{1-p-p \lambda_{2}}
$$

and are satisfied when

$$
p<\frac{1}{1+\lambda_{i}} \quad \text { for every } i=1,2 .
$$

As shown in the proof above, $\mathcal{V}_{i}^{G L}$ is convex in $r_{i}$ for every $i=1,2$. This follows not from the model we propose, but from the definition of $G L$. Accordingly, it is intuitive that there exist three types of extremal equilibria: The presence of a breakdown probability and loss aversion creates a trade-off for the agents, and the convexity of $\mathcal{V}_{i}^{G L}$ leads to these extremal equilibria. ${ }^{6}$

Proposition 1 indicates the multiplicity of Nash equilibria. As illustrated in Fig. 2, all relevant inequalities can be summarized on an interval for the breakdown probability, $p \in[0,1]$. Without loss of generality, assume that $\lambda_{1}>\lambda_{2}$. In case $\mathrm{D}$, only the equilibrium (i) is realized. In case $C$, only the equilibrium (ii)(a) is realized. In case B, the equilibria (ii)(a) and (iii) are realized. In case A, the equilibria (ii)(a), (ii)(b), and (iii) are realized. For the sake of completeness, we should note that if $2 \lambda_{2}>\lambda_{1}$, then case $\mathrm{C}$ disappears as a result of either case $\mathrm{B}$ expanding rightwards or case $\mathrm{D}$ expanding leftwards.

The comparative statics for the corner equilibrium on the disagreement point outcome, i.e., $r^{*}=\left(d_{1}, d_{2}\right)$, is quite obvious. Its existence depends only on $p, \lambda_{1}$, and $\lambda_{2}$. In particular, for sufficiently low values of these parameters, there exists no such equilibrium. The intuition for this result is: as the values of $p, \lambda_{1}$, or $\lambda_{2}$ decrease, the trade-off generated by the presence of disagreement possibility and loss aversion becomes smaller. In other words, the factors that encourage an agent to choose a low reference point are weak. In such a case, agent $i$ 's best response to some $r_{j}$ would be to choose $r_{i}=1-r_{j}$. Accordingly, the strategy profile $\left(d_{1}, d_{2}\right)$ cannot be realized as an equilibrium.

Focusing on the limit cases for the breakdown probability $p$, it is easy to see that when $p=1$, case $\mathrm{D}$ occurs with a unique equilibrium on the disagreement point outcome. This follows because with such an extreme breakdown probability, $G L$ will never be used, so that the agents protect themselves from falling in the loss domain by lowering their reference point outcomes as much as possible. Conversely, when $p=0$, there is no risk of breakdown so that $G L$ will always be employed. Hence, only the advantages from a reference point exist, i.e., the trade-off disappears. Accordingly, both agents prefer higher reference point outcomes, and as a result case A occurs with multiple equlibria on the frontier. Focusing on the limit cases for the loss aversion coefficient $\lambda_{i}$, one can see in Fig. 2 that case D disappears when $\lambda_{2}$ approaches 0 ,

\footnotetext{
6 To the best of our knowledge, there is only one alternative to $G L$, which is the tempered aspirations solution proposed by Balakrishnan et al. (2011). This solution concept is similar to $G L$ in that it also employs a reference point. An important observation for our purposes is that the utility it yields to an agent is also convex in the agent's reference point. Hence, we think that $G L$ and its alternative would lead to qualitatively similar results.
} 


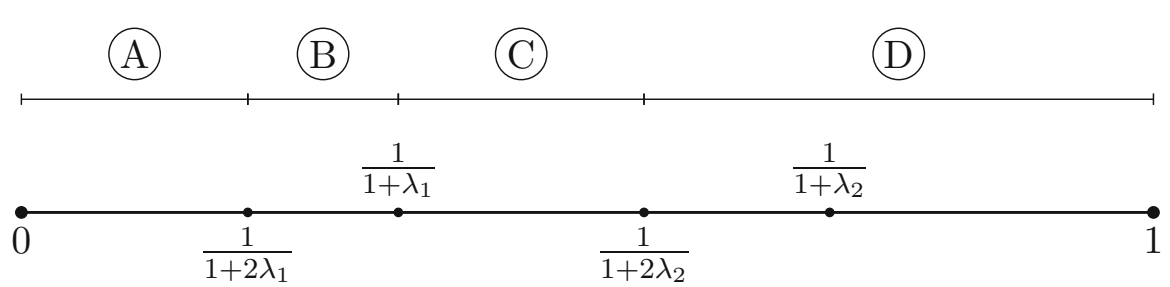

Fig. 2 Critical values for the breakdown probability $p$ (assuming $\lambda_{1}>\lambda_{2}$ )

whereas cases A and B both disappear when $\lambda_{1}$ approaches $\infty$. Intuitively, a lower $\lambda_{i}$ decreases the cost of having a high reference point outcome so that the agents tend to choose a higher reference point outcome as they become less loss averse. Finally, if $p, \lambda_{1}$, and $\lambda_{2}$ all approach 0 , then our model converges to a divide-the-dollar game (with an outside option), since any reference point outcome pair $\left(r_{1}^{*}, 1-r_{1}^{*}\right)$ satisfying $d_{1} \leq r_{1}^{*} \leq 1-d_{2}$ would be a Nash equilibrium, having a strong similarity with the set of Nash equilibria of that game.

Can one benefit from being loss averse? Remark 1 answers this question: in short, the answer is "no".

Remark 1 Assume that $\lambda_{1}>\lambda_{2}$. As seen in Fig. 2, there are values of the breakdown probability for which the strategy profile $\left(d_{1}, 1-d_{1}\right)$ is an equilibrium, whereas the strategy profile $\left(1-d_{2}, d_{2}\right)$ is not. The converse is not true. This shows a clear disadvantage for the more loss averse agent.

The size of the set of equilibrium reference point outcomes on the frontier depends on more parameters. Remark 2 summarizes comparative static results on this set of equilibria. It is worth noting here that these results are found by taking the derivatives of the lower and upper bounds of the equilibrium interval with respect to the corresponding model parameters.

Remark 2 Assume that $p\left(1+\lambda_{i}\right)<1$ for both $i=1,2$. Then the interval for $r_{1}^{*}$ narrows down from below if

(i) $p$ increases,

(ii) (a) $d_{1}$ increases when $p\left(1+2 \lambda_{1}\right)<1$,

(b) $d_{1}$ decreases when $p\left(1+2 \lambda_{1}\right)>1$,

(iii) $d_{2}$ decreases, or

(iv) $\lambda_{1}$ increases.

And the interval for $r_{1}^{*}$ narrows down from above if

(i) $p$ increases,

(ii) $d_{1}$ decreases,

(iii) (a) $d_{2}$ increases when $p\left(1+2 \lambda_{2}\right)<1$,

(b) $d_{2}$ decreases when $p\left(1+2 \lambda_{2}\right)>1$, or

(iv) $\lambda_{2}$ increases.

The intuitions for the comparative static results on $p, \lambda_{1}$, and $\lambda_{2}$ are similar to those in the comparative static analyses on $r^{*}=d$ equilibrium. As their values decrease, the 
equilibrium on the disagreement point outcome is more likely to vanish, whereas we observe more equilibria on the frontier. Furthermore, the effects of $d_{1}$ and $d_{2}$ on this set of equilibria depend on whether $\left(d_{1}, d_{2}\right)$ is realized as an equilibrium or not. If so, a decrease in $d_{1}$ or $d_{2}$ narrows down the set of equilibria on the frontier. This is because when $p, \lambda_{1}$, and $\lambda_{2}$ are above their critical levels, a decrease in the disagreement point outcome is more costly due to loss aversion. If otherwise, the respective effect might be mixed. More precisely, when $p\left(1+2 \lambda_{i}\right)<1$ for some $i=1,2$, an increase in $d_{i}$ shifts the interval but rather asymmetrically.

Remark 3 provides a sufficient condition for the uniqueness of the equilibrium on the frontier, as well as a necessary condition for its existence.

Remark 3 Assume that $p\left(1+\lambda_{i}\right)<1$ for both $i=1,2$. The equilibrium on the frontier is unique, if

$$
\frac{\lambda_{1}}{1-p-p \lambda_{1}}+\frac{\lambda_{2}}{1-p-p \lambda_{2}}=\frac{1}{p} .
$$

Furthermore, there exists no such equilibrium if the left-hand-side is greater than the right-hand-side.

\section{Concluding remarks}

We set up a bilateral bargaining model with a reference point and allow this reference point to be endogenously and strategically determined by the agents prior to the resolution of conflict. In our model, two loss averse agents simultaneously choose their reference point outcomes, which can also be interpreted as their commitment levels. While making these decisions, both agents know that with a certain probability they will not be able to reach an agreement and will receive their disagreement outcomes, whereas with the remaining probability the conflict will be resolved by an arbitrator using the Gupta-Livne solution. The presence of such an uncertainty creates a tradeoff in the determination of the reference point outcomes. As a result, the equilibrium reference point outcomes depend on the breakdown probability as well as both agents' loss aversion coefficients and disagreement point outcomes. Our model offers intuitive comparative statics on these parameters.

In our model, we take the breakdown probability as exogenously given. Endogenizing this parameter is an interesting venue for future research. In fact, such an endogenous breakdown probability may yield important insights even when agents have standard preferences. Finally, it is worthwhile saying that we presented one reasonable method for endogenizing reference points in cooperative bargaining problems. In this paper, reference point outcomes are strategically determined in a pre-bargaining game. To the best of our knowledge, this is the first attempt along these lines. Future research may present alternative methods.

Acknowledgements We would like to thank two anonymous reviewers and an associate editor for their constructive comments, which improved the paper. Emin Karagözoğlu acknowledges financial support from Bilim Akademisi (The Science Academy, Turkey). 


\section{References}

Ashenfelter O, Bloom DE (1984) Models of arbitrator behavior: theory and evidence. Am Econ Rev 74:111124

Balakrishnan PV, Gómez JC, Vohra RV (2011) The tempered aspirations solution for bargaining problems with a reference point. Math Soc Sci 62:144-150

Bartling B, Schmidt KM (2015) Reference points, social norms, and fairness in contract renegotiation. J Eur Econ Assoc 13:98-129

Bazerman MH (1985) Norms of distributive justice in interest arbitration. Ind Labor Relat Rev 38:558-570

Blount S, Thomas-Hunt MC, Neale MA (1996) The price is right—or is it? A reference point model of two-party price negotiations. Organ Behav Hum Decis Process 68:1-12

Bolton GE, Karagözoğlu E (2016) On the influence of hard leverage in a soft leverage bargaining game: the importance of credible claims. Games Econ Behav 99:164-179

Bozbay I, Dietrich F, Peters H (2012) Bargaining with endogenous disagreement: the extended KalaiSmorodinsky solution. Games Econ Behav 74:407-417

Brito DL, Buoncristiani A, Intriligator MD (1977) A new approach to the Nash bargaining problem. Econometrica 45:1163-1172

Compte O, Jehiel P (2004) Bargaining with reference dependent preferences. Mimeo, Paris School of Economics

Delfour MC (2012) Introduction to optimization and semidifferential calculus. Society for Industrial and Applied Mathematics, Philadelphia

Driesen B, Perea A, Peters H (2011) The Kalai-Smorodinsky bargaining solution with loss aversion. Math Soc Sci 61:58-64

Driesen B, Perea A, Peters H (2012) Alternating offers bargaining with loss aversion. Math Soc Sci 64:103118

Ellingsen T, Miettinen T (2008) Commitment and conflict in bilateral bargaining. Am Econ Rev 98:16291635

Ellingsen T, Miettinen T (2014) Tough negotiations: bilateral bargaining with durable commitments. Games Econ Behav 87:353-366

Gächter S, Riedl A (2005) Moral property rights in bargaining with infeasible claims. Manag Sci 51:249-263

Gupta S, Livne ZA (1988) Resolving a conflict situation with a reference outcome: an axiomatic model. Manag Sci 34:1303-1314

Hyndman K (2011) Repeated bargaining with reference-dependent preferences. Int J Game Theory 40:527549

Kahneman D, Tversky A (1979) Prospect theory: an analysis of decision under risk. Econometrica 47:263291

Karagözoğlu E, Riedl A (2015) Performance information, production uncertainty, and subjective entitlements in bargaining. Manag Sci 61:2611-2626

Köszegi B, Rabin M (2006) A model of reference-dependent preferences. Q J Econ 121:1133-1165

Kristensen H, Gärling T (2000) Anchor points, reference points, and counteroffers in negotiations. Group Decis Negot 9:493-505

Luce D, Raiffa H (1957) Games and decisions. Wiley, New York

Muthoo A (1992) Revocable commitment and sequential bargaining. Econ J 102:378-387

Nash JF (1953) Two-person cooperative games. Econometrica 21:128-140

Sarver T (2012) Optimal reference points and anticipation. Working paper, Duke University

Shalev J (2000) Loss aversion equilibrium. Int J Game Theory 29:269-287

Shalev J (2002) Loss aversion and bargaining. Theor Decis 52:201-232

Thomson W (1981) A class of solutions to the bargaining problem. J Econ Theory 25:431-442

Vartiainen H (2007) Collective choice with endogenous reference outcome. Games Econ Behav 58:172-180 